\title{
Naturally occurring NAD-independent Haemophilus parainfluenzae
}

\author{
ROSA GromKova* AND HENDRIK KOORNHOF \\ Emergent Pathogen Research Unit of the South African Medical Research Council, School of Pathology of the \\ University of the Witwatersrand and the South African Institute for Medical Research, 7 York Road, \\ Parktown, Johannesburg 2193, South Africa
}

(Received 5 September 1989; revised 8 December 1989; accepted 2 February 1990)

\begin{abstract}
Four, NAD-independent, clinical isolates of Haemophilus parainfluenzae were recovered from a genital ulcer, a purulent skin lesion, a sputum specimen and a throat swab respectively. With the exception of NAD requirement, the strains exhibited the biochemical characteristics of $\boldsymbol{H}$. parainfluenzae biotype II. The genetic relationship between these isolates and a standard strain of $\boldsymbol{H}$. parainfluenzae was determined by testing transforming activities of two chromosomal markers, streptomycin resistance and nalidixic acid resistance. The clinical isolates were efficient donors and recipients in transformation. In addition, we demonstrated transfer of the genes conferring NAD independence to typical, NAD-requiring $H$. parainfluenzae and Haemophilus influenzae strains.
\end{abstract}

\section{Introduction}

The laboratory identification and the classification of species of the genus Haemophilus is based on their requirement for either NAD (V-factor) or haemin (Xfactor) or both. Haemophilus parainfluenzae is NADdependent, while Haemophilus influenzae requires both NAD and haemin. According to the present taxonomic criteria bacteria that are able to grow in the absence of NAD cannot be classified as $H$. parainfluenzae or $H$. influenzae. The NAD dependence is considered to be a genetically stable property because of the inability of these organisms to revert to NAD independence. Attempts to obtain by DNA transformation strains of $H$. parainfluenzae that do not require NAD have been unsuccessful (White \& Granick, 1963) and the isolation of such strains from clinical specimens has not been reported. Man is the natural host of $H$. parainfluenzae and $H$. influenzae and these species are usually present in healthy individuals. $H$. parainfluenzae is a known but occasional cause of endocarditis, pneumonia and meningitis while $H$. influenzae is a major pathogen especially in children, causing serious infections such as meningitis, epiglottitis, pneumonia and septicaemia. In this paper we describe the biochemical and genetic properties of four clinical isolates from unrelated sources that had the usual features of $H$. parainfluenzae but were NADindependent.

\footnotetext{
* Correspondence should be sent to PO Box 1038, Johannesburg 2000 , South Africa.
}

\section{Methods}

Bacterial strains and growth. Three unusual strains were isolated from paediatric patients at Baragwanath Hospital, Johannesburg whilst examining $20 \beta$-lactamase-producing Haemophilus-like bacteria. These strains were isolaied from a sputum sample, a specimen of pus and a throat swab, and were designated strains 9,15 and 19 respectively. In addition, we recovered another isolate, designated strain 78 , together with a typical $H$. parainfluenzae strain, designated strain 79 , from a genital ulcer in a male patient attending a clinic for sexually transmitted diseases at Carletonville, near Johannesburg. $H$. influenzae $\mathrm{Rd}$ and $H$. parainfluenzae 14 (Boss) were provided by G. Leidy, Columbia University, New York, USA. Antibiotic-resistant strains, used as donors in DNA transformation, were obtained by selecting spontaneous mutants on antibiotic-containing plates. $H$. influenzae $\mathrm{Rd}$ was grown in Brain Heart Infusion (BHI) broth and BHI agar (Becton Dickinson) supplemented with $10 \mu \mathrm{g}$ haemin $\mathrm{ml}^{-1}$ and $2 \mu \mathrm{g} \mathrm{NAD} \mathrm{ml} \mathrm{N}^{-1}$ (both from Sigma). For growth of $H$. parainfluenzae the BHI medium was supplemented with $2 \mu \mathrm{g} \mathrm{NAD} \mathrm{ml}^{-1}$.

Biochemical characterization. The biochemical properties of the four NAD-independent isolates were compared with those of typical strains of $H$. parainfluenzae and Haemophilus aphrophilus according to the scheme proposed by Kilian (1976).

$\beta$-Lactamase production. The nitrochephin method was used to demonstrate $\beta$-lactamase production (O'Callaghan et al., 1974).

DNA transformation. Purified DNA was prepared by the method described by Marmur (1961). Competence was induced by the static aerobic procedure (Gromkova \& Goodgal, 1979; Gromkova et al., 1989). For the transformation assay $0.1 \mathrm{ml} \mathrm{DNA}$ at a saturating concentration of $1 \mu \mathrm{g} \mathrm{ml}^{-1}$ was added to $0.8 \mathrm{ml}$ BHI broth. After the addition of $0.1 \mathrm{ml}$ competent cells, the mixture was incubated for $30 \mathrm{~min}$ at $35^{\circ} \mathrm{C}$ without shaking and then pancreatic DNAase (Sigma) was added to $10 \mu \mathrm{g} \mathrm{ml}^{-1}$. After a further $10 \mathrm{~min}$ incubation at $35^{\circ} \mathrm{C}$, the mixture was diluted and each dilution was plated in triplicate for transformants using an overlay method. For expression of the genes 
conferring NAD independence, the transformation mixture was incubated for $3 \mathrm{~h}$ at $35^{\circ} \mathrm{C}$. Each transformation experiment was repeated three times.

\section{Results}

\section{Growth and biochemical characteristics}

Strains 9, 15, 19 and 78 were able to grow aerobically on chocolate agar and on $5 \%(\mathrm{v} / \mathrm{v})$ horse blood agar. On the latter medium they produced green discolouration similar to that of $\alpha$-haemolytic streptococci. The colonies on chocolate agar were small (about $2 \mathrm{~mm}$ in diameter) and flat with smooth and shiny surfaces. They exhibited a striking similarity to the colonies of $H$. parainfluenzae 79 and $H$. parainfluenzae 14 . They also produced a smell characteristic of $H$. parainfluenzae. However, unlike $H$. parainfluenzae, strains $9,15,19$ and 78 were able to grow on blood agar and in BHI broth and BHI agar lacking NAD. This property was maintained when three different batches of BHI agar were used and when 20 single colony passages on unsupplemented BHI agar were done. The disc test for $\mathrm{V}$-factor requirement on $\mathrm{BHI}$ agar was also negative. The four clinical isolates were all able to produce the satellite effect (Kilian, 1976) when streaked on unsupplemented BHI agar inoculated with a typical NAD-requiring $H$. parainfluenzae strain 14 . This suggests that the NAD independence was probably due to the ability of the clinical isolates to synthesize and release into the medium either NAD or some other metabolite that may also serve as a $\mathrm{V}$-factor such as NAD-phosphate, nicotinamide mononucleotide or nicotinamide riboside.

Gram-stained preparations examined microscopically showed small Gram-negative rods arranged in short or long chains.

The properties of the clinical isolates were compared with those of $H$. parainfluenzae and $H$. aphrophilus. The results presented in Table 1 show that the only feature that distinguished the clinical isolates from $H$. parainfluenzae strains was NAD requirement. The remaining biochemical reactions corresponded to biotype II of $H$. parainfluenzae. This pattern was clearly distinguishable from that of $H$. aphrophilus, which does not require NAD for growth. Unlike the clinical isolates, $H$. aphrophilus is characteristically urease-negative, ornithine-decarboxylase- and hydrogen-sulphide-negative and its growth is stimulated by $\mathrm{CO}_{2}$. The four NAD-independent isolates all produced $\beta$-lactamase, a property that typical $H$. parainfluenzae may also acquire.

\section{Transformation studies}

Two chromosomal genes conferring resistance to streptomycin and nalidixic acid respectively were used as markers in transformation experiments. The ribosomal streptomycin-resistance ( $\mathrm{Str}^{\mathrm{r}}$ ) gene is considered to be more conserved among species than the non-ribosomal, nalidixic-acid-resistance ( $\mathrm{Nal}^{\mathrm{r}}$ ) gene (Dubnau et al., 1965). Both these genes are located on the chromosome. Double-resistant mutants $\left(\mathrm{Str}^{\mathrm{r}}\right.$ and $\mathrm{Nal}^{\mathrm{r}}$ ) were selected from each of the four NAD-independent clinical isolates and from the $H$. parainfluenzae strains. The highly transformable strain $H$. parainfluenzae 14 (Leidy et al., 1956) was employed as recipient. The results in Table 2 show that $\mathrm{Str}^{\mathrm{r}}$ and $\mathrm{Nal}^{\mathrm{r}}$ markers from the NADindependent clinical isolates were highly efficient in transforming $H$. parainfluenzae 14 . There was a two- to threefold difference betweeen the efficiencies of transformation of these markers and the $\mathrm{Str}^{\mathrm{r}}$ and $\mathrm{Nal}^{\mathrm{r}}$ markers of the isogenic $H$. parainfluenzae 14 donor. The transforming activity of DNA from the NAD-independent donors was equal to that of the $H$. parainfluenzae 79 strain from South Africa. The results were reproducible when repeated three times with different batches of competent cells. The results of a reciprocal transformation experiment are given in Table 3. All strains were good recipients but showed differences in their levels of competence, strain 78 being a more efficient recipient than the other NAD-independent isolates. Strain variation in competence levels has been observed in several transformation systems including Haemophilus species (Rowji et al., 1989).

As the successful transfer of genes conferring NAD independence in Haemophilus had not been reported previously, we investigated the transfer of such genes using the NAD-independent isolates as the source of donor DNA with both $H$. parainfluenzae 14 and $H$. influenzae $\mathrm{Rd}$ as recipients. $H$. influenzae $\mathrm{Rd}$ is a highly transformable strain that has been used extensively in transformation studies (Alexander \& Leidy, 1956). Since neither the location of the NAD genes nor their transforming ability was known, the transforming efficiency of chromosomal markers was used to determine the level of competence in the recipient cells. We were able to demonstrate transformation to NAD independence in both recipients. The frequency of transformation by the NAD genes in $H$. parainfluenzae 14 (Table 2) was higher than in $H$. influenzae $\mathrm{Rd}$ (Table 4), probably due to the degree of genetic homology with donor DNA. The efficiency of transformation of the NAD marker in $H$. parainfluenzae 14 was about 10 - to 20 fold lower than that of the chromosomal Str $^{\mathrm{r}}$ marker whereas in the $H$. influenzae $\mathrm{Rd}$ recipient these two markers exhibited similar transformation frequencies. 
Table 1. Characteristics of unusual clinical isolates and typical $H$. parainfluenzae and $H$. aphrophilus strains

Tests were done acording to the techniques recommended by Kilian (1976).

For designation of strains see Methods.

\begin{tabular}{lccc}
\hline & & & $\begin{array}{c}\text { Unusual } \\
\text { strains }\end{array}$ \\
& $\begin{array}{c}\text { H. parainfluenzae } \\
\text { strains } 14,79\end{array}$ & H. aphrophilus* & $9,15,19,78$ \\
\hline NAD requirement & + & - & - \\
Porphyrin & + & + & + \\
Indole & - & - & - \\
Ornithine decarboxylase & + & - & + \\
Hydrogen sulphide & + & - & + \\
Urease & + & - & + \\
Oxidase & + & + & + \\
Alkaline phosphatase & + & + & + \\
Nitrate reduction & + & + & - \\
Lactose, acid & - & + & + \\
Glucose, acid & + & + & - \\
Sucrose, acid & + & - & + \\
CO $\mathrm{O}_{2}(5 \%)$ enhances growth & - & & + \\
\hline \hline
\end{tabular}

${ }^{*} H$. aphrophilus according to Kilian (1976).

Table 2. Transformation of $H$. influenzae 14 by DNA from $N A D$-independent strains

The concentrations of streptomycin and nalidixic acid used for selection of transformants were $100 \mu \mathrm{g} \mathrm{ml}^{-1}$ and $3 \mu \mathrm{g} \mathrm{ml}^{-1}$ respectively. For designation of strains see Methods.

\begin{tabular}{|c|c|c|c|}
\hline \multirow{2}{*}{$\begin{array}{l}\text { DNA } \\
\text { donor }\end{array}$} & \multicolumn{3}{|c|}{$\begin{array}{l}\text { No. of transformants } \\
\left(\text { per } 5 \times 10^{8} \text { c.f.u. } \mathrm{ml}^{-1}\right)\end{array}$} \\
\hline & Str ${ }^{r}$ & $\mathrm{Nal}^{\mathrm{r}}$ & NAD independence \\
\hline Strain 78 & $2 \cdot 1 \times 10^{6}$ & $4.5 \times 10^{6}$ & $9.8 \times 10^{4}$ \\
\hline Strain 15 & $1.3 \times 10^{6}$ & $3.3 \times 10^{6}$ & $6.9 \times 10^{4}$ \\
\hline Strain 9 & $1.7 \times 10^{6}$ & $2.4 \times 10^{6}$ & $7 \cdot 5 \times 10^{4}$ \\
\hline Strain 19 & $2 \cdot 1 \times 10^{6}$ & $3.9 \times 10^{6}$ & $8 \cdot 1 \times 10^{4}$ \\
\hline H. parainfluenzae 79 & $2.2 \times 10^{6}$ & $4 \cdot 1 \times 10^{6}$ & 0 \\
\hline H. parainfluenzae 14 & $4 \cdot 1 \times 10^{6}$ & $6.8 \times 10^{6}$ & 0 \\
\hline
\end{tabular}

Table 3. Reciprocal transformation of NAD-independent strains and of $H$. parainfluenzae 14 and 79

For designation of strains see Methods.

\begin{tabular}{lcc}
\hline \hline & \multicolumn{1}{c}{$\begin{array}{c}\text { No. of transformants* } \\
\text { (per } 5 \times 10^{8} \text { c.f.u. } \mathrm{ml}^{-1} \text { ) }\end{array}$} \\
\cline { 2 - 3 } \multicolumn{1}{c}{ Recipient } & $\begin{array}{c}\text { H. parainfluenzae } 14 \\
\text { donor DNA }\end{array}$ & Isogenic donor DNA \\
\hline Strain 78 & $2.1 \times 10^{6}$ & $3.9 \times 10^{6}$ \\
Strain 15 & $3.1 \times 10^{5}$ & $4.2 \times 10^{5}$ \\
Strain 9 & $2.2 \times 10^{5}$ & $3.1 \times 10^{5}$ \\
Strain 19 & $3.2 \times 10^{5}$ & $4.8 \times 10^{5}$ \\
H. parainfluenzae 79 & $2.2 \times 10^{6}$ & $4.2 \times 10^{6}$ \\
H. parainfluenzae 14 & $4.9 \times 10^{6}$ & \\
\hline \hline
\end{tabular}

* See Table 2 legend.
Table 4. Transforming activity of the NAD independence and $S t r$ markers in $H$. influenzae $R d$ as recipient

For designation of strains see Methods.

\begin{tabular}{lcc}
\hline & \multicolumn{2}{c}{$\begin{array}{c}\text { No. of transformants* } \\
\text { (per 5 } 510^{8} \text { c.f.u. } \mathbf{m}^{-1} \text { ) }\end{array}$} \\
\cline { 2 - 3 } \multicolumn{1}{c}{ DNA donor } & $\begin{array}{c}\text { NAD } \\
\text { independence }\end{array}$ & Str $^{\mathrm{r}}$ \\
\hline Strain 78 & $3 \times 10^{3}$ & $2.2 \times 10^{3}$ \\
Strain 15 & $2.2 \times 10^{3}$ & $2.5 \times 10^{3}$ \\
Strain 9 & $1.5 \times 10^{3}$ & $1.9 \times 10^{3}$ \\
Strain 19 & $1.1 \times 10^{3}$ & $1.5 \times 10^{3}$ \\
H. parainfluenzae 79 & 0 & $2.2 \times 10^{3}$ \\
H. parainfluenzae 14 & 0 & $2.8 \times 10^{3}$ \\
H. influenzae Rd & 0 & $3.8 \times 10^{6}$ \\
\hline . & &
\end{tabular}

* See Table 2 legend.

\section{Discussion}

NAD is an essential coenzyme for energy-yielding processes in both eukaryotic and prokaryotic cells. Two major biosynthetic pathways have been described. One involves de novo synthesis of NAD and the other is based on the utilization of preformed pyridine bases obtained from the breakdown of intracellular NAD or from exogenous sources (Foster \& Moat, 1980). It would be of interest to study which of these pathways is used for the synthesis of NAD in the NAD-independent $H$. parainfluenzae strains. Previous studies have shown that the NAD-independent species Haemophilus haemoglobinophilus synthesizes NAD from nicotinamide but is incapable of de novo NAD synthesis (Kassarov \& Moat, 1973). 
Requirements for NAD and haemin have been considered for many years to be essential criteria for the classification of Haemophilus species and have also formed the basis for their laboratory identification. This emphasis on growth factors may well explain why NADindependent $H$. parainfluenzae strains have not been recognized earlier by clinical microbiologists.

The finding that, with the exception of NAD independence, all 12 biochemical reactions were identical to those of biotype II of $H$. parainfluenzae (Table 1) and that two chromosomal genes (one ribosomal and one non-ribosomal) were highly efficient in transforming a standard strain of $H$. parainfluenzae (Table 2) strongly suggests that the isolates are $H$. parainfluenzae. Because of their ability to grow in the absence of NAD, consideration should be given to assign the four isolates to a new biotype. The two- to threefold differences between the transforming efficiencies of isogenic $H$. parainfluenzae 14 DNA and those of DNA from the clinical isolates were probably due to genetic heterogeneity within the species (Table 2). Similar observations have been made previously when the properties of different $H$. parainfluenzae strains have been compared (Pohl, 1981). Recently two former members of the genus Haemophilus - Haemophilus pleuropneumoniae and Haemophilus avium - were transferred to the genera Actinobacillus and Pasteurella, respectively (Pohl et al., 1983). This was based on genetic homology and phenotypic considerations in which the NAD requirement was shown not to be of overriding importance. Furthermore, Pohl et al. (1983) demonstrated that some $H$. pleuropneumoniae strains do not require NAD for growth. Based on these developments and our findings we agree with Pohl (1981) and Pohl et al. (1983) that new criteria be formulated for the classification and laboratory identification of this important taxonomic group. An identification scheme based on that proposed by Kilian (1976) would be appropriate. $H$. influenzae strains that are NAD-independent should constitute a new biotype. At present, the only other Haemophilus species that does not require either haemin or NAD is $H$. aphrophilus; this organism can be distinguished from $H$. parainfluenzae by its ability to ferment lactose and deoxyribose and to produce oxidase. In addition, unlike $H$. parainfluenzae, $H$. aphrophilus fails to produce $\mathrm{H}_{2} \mathrm{~S}$ and its growth is stimulated by $\mathrm{CO}_{2}$. One should also bear in mind that atypical NAD-independent strains of $\boldsymbol{H}$. parainfluenzae are able to grow on media such as blood agar and BHI agar that usually do not support the growth of typical $H$. parainfluenzae. The recovery of NAD-independent strains from natural sources demands that NAD requirement should not be considered a predominant taxonomic criterion for this species.

Previous studies have shown that there is no correla- tion between marker efficiencies in homospecific as opposed to heterospecific transformation in the genus Haemophilus (Goodgal \& Gromkova, 1973). In both $H$. influenzae $\mathrm{Rd}$ and $H$. parainfluenzae 14 recipients, marker efficiencies in transformation with homologous chromosomal DNA do not generally vary more than by a factor of two or three. The reason for the bigger differences in the transforming efficiencies of the NAD and $\operatorname{Str}^{\mathrm{r}}$ markers in $H$. parainfluenzae 14 is unknown. However, several explanations can be offered: (i) incomplete sequence homology between donor and recipient DNA; (ii) poor expression of the NAD genes probably due to changes of some regulatory proteins; (iii) bigger size of the NAD marker relative to that of the Str ${ }^{r}$ marker.

The similarity of transformation efficiencies of NAD and $\operatorname{Str}^{\mathrm{r}}$ markers in heterospecific transformation (see Table 4) does not necessarily indicate that the sizes of these two markers are the same in homospecific transformation (Table 2). Previous studies have shown that DNA fragments that are biologically active in heterospecific transformation may not be able to transform homospecifically, suggesting lack of correlation between the sizes of the biologically active genetic markers (Goodgal \& Gromkova, 1973). Furthermore, other factors besides sequence homology and the size of the markers have been shown to play a role in heterospecific transformation in Haemophilus species (Steinhart \& Herriott, 1968; Goodgal \& Gromkova, 1973). The similar frequencies of NAD and $\mathrm{Str}^{\mathrm{r}}$ transformation in $H$. influenzae, therefore, does not necessarily indicate similarity between sizes of these two markers. Another explanation of the lower efficiency of NAD transformation of $H$. parainfluenzae recorded here is the possibility of extrachromosomal location of genes conferring NAD independence. It has been previously demonstrated that plasmid transformation in $H$. parainfluenzae is less efficient than by chromosomal DNA (Gromkova \& Goodgal, 1979).

The demonstration that typical NAD-requiring strains of $H$. parainfluenzae and $H$. influenzae can be transformed to NAD independence under experimental conditions may have biological significance. $H$. influen$z a e$ and $H$. parainfluenzae share a common ecological niche and both species are naturally transformable. Furthermore, they are able to undergo spontaneous autolysis followed by the release of DNA. This suggests that DNA-mediated transfer may occur in vivo. One can speculate that the genes conferring NAD-independence to $H$. parainfluenzae have been acquired by genetic exchange with an organism able to synthesize NAD in a shared ecological milieu. The finding that the NADindependent $H$. parainfluenzae strains were able to develop competence suggests that the NAD genes may 
have been acquired by DNA transformation. However, other mechanisms of gene transfer cannot be excluded. Although not impossible, it is unlikely that the NADindependent clinical isolates have been generated by a mutation. Attempts to obtain such mutants in vitro have been unsuccessful (R. Gromkova, unpublished results). Since we were able to demonstrate in vitro transfer of the genes conferring NAD independence to $H$. influenzae by DNA transformation, it is possible that the latter, as is the case with $H$. parainfluenzae, may also occur in nature. Because of the greater clinical significance of $H$. influenzae, it is important to be aware of such a possibility and to use diagnostic procedures that cater for the identification of NAD-independent clinical isolates.

We wish to thank G. Leidy, Y. Dangor, C. Heney and J. Summer for kindly providing us with bacterial strains.

\section{References}

Alexander, H. E. \& Leidy, E. (1955). Origin of species in microorganisms : use of transformation as a tool. American Journal of Diseases of Children 90, 560-561.

Dubnau, D., Smith, I., Morell, P. \& Marmur, J. (1965). Gene conservation in bacillus species. I. Conserved genetic and nucleic acid base sequence homologies. Proceedings of the Society for Experimental Biology and Medicine 54, 491-498.

Foster, J. \& MOAT, A. (1980). Nicotinamide adenine dinucleotide biosynthesis and pyridine nucleotide cycle metabolism in microbiological systems. Microbiological Reviews 44, 83-105.

GoodGal, S. H. \& Gromkova, R. (1973). The biological specificity of Haemophilus endodeoxyribonucleases which attack heterologous DNA. In Bacterial Transformation, pp. 743-758. Edited by L. Archer. London \& New York: Academic Press.
Gromkova, R. \& Goodgal, S. H. (1979). Transformation by plasmid and chromosomal DNA in Haemophilus parainfluenzae. Biochemical and Biophysical Research Communications 88, 1428-1431.

Gromkova, R., RowJI, P. B. \& KoorNHoF, H. J. (1989). Induction of competence in nonencapsulated and encapsulated strains of Haemophilus influenzae. Current Microbiology 19, 241-245.

KASAROV, L. B. \& MOAT, A. (1973). Biosynthesis of nicotinamide adenine dinucleotide in Haemophilus haemoglobinophilus. Biochimica et Biophysica Acta 320, 372-378.

KILIAN, M. (1976). A taxonomic study of the genus Haemophilus with the proposal of a new species. Journal of General Microbiology 93, 9-63.

Leidy, G., Hahn, E. \& AlEXander, H. E. (1956). On the specificity of the desoxyribonucleic acid which induces streptomycin resistance in Haemophilus. Journal of Experimental Medicine 104, 305-320.

MARMUR, J. (1961): A procedure for the isolation of deoxyribonucleic acid from microorganisms. Journal of Molecular Biology 3, 208-218.

O'Callaghan, C. H., Morris, A., Kirby, S. M. \& Shingler, A. H. (1974). Novel method for detection of beta-lactamases by using a chromogenic cephalosporin substrate. Antimicrobial Agents and Chemotherapy 1, 283-288.

PoHL, S. (1981). DNA relatedness among members of Haemophilus, Pasteurella and Actinobacillus. In Haemophilus, Pasteurella and Actinobacillus, pp. 245-253. Edited by M. Kilian, W. Frederiksen \& E. L. Biberstein. London: Academic Press.

Pohl, S., Bertschinger, H. U., Frederiksen, W. \& ManNheim, W. (1983). Transfer of Haemophilus pleuropneumoniae and the Pasteurella haemolitica-like organism causing porcine necrotic pleuropneumonia to the genus Actinobacillus (Actinobacillus pleuropneumoniae comb. nov.) on the basis of phenotypic and deoxyribonucleic acid relatedness. International Journal of Systematic Bacteriology 33, 510514.

Rowj, P., Gromkova, R. \& KoornhoF, H. (1989). Genetic transformation in encapsulated clinical isolates of Haemophilus influenzae type b. Journal of General Microbiology 135, 2775-2782.

SteinharT, W. L. \& Herriot, R. M. (1968). Genetic integration in the heterospecific transformation of Haemophilus influenzae cells by Haemophilus parainfluenzae deoxyribonucleic acid. Journal of Bacteriology 96, 1725-1731.

WhITE, D. \& GraNick, S. (1963). Hemin biosynthesis in Haemophilus. Journal of Bacteriology 85, 842-850. 